\title{
Research on Mine Gas Monitoring System Based on Single-chip Microcomputer
}

\author{
Shusen Meng \\ Jiangsu Vocational Institute of Commerce, Nanjing, Jiangsu Province, China \\ mengshusen@126.com
}

Keywords: Single-chip microcomputer, Mine, Gas monitoring, Monitoring system

\begin{abstract}
With the continuous development of the coal industry, mine gas accidents have increasingly become an important factor affecting coal mine safety. In major coal mine accidents, gas accidents account for about $70 \%$ to $80 \%$. There are many reasons for gas accidents, but the most essential reason is the formation of gas accumulation. Only by avoiding the accumulation of gas can the gas accident be eliminated fundamentally. The most important thing to avoid gas accumulation is to discover gas accumulation in time and deal with it in time. This must rely on various gas monitoring systems. This paper proposes a mine-based gas monitoring system based on single-chip microcomputer. Through a brief introduction to the system, it is hoped that the occurrence of gas accidents can be effectively controlled.
\end{abstract}

\section{Introduction}

In recent years, with the rapid development of China's economy, the consumption of coal has continued to grow. Especially in recent years, the price of coal has been increasing, and higher requirements have been placed on the output of coal [1]. However, in actual coal production, mine accidents continue to occur. The number of mine deaths per year in China's coal production is nearly 10,000 . Therefore, we have to pay more attention to the safety of coal production. There are many reasons for mine disasters, and mine accidents caused by gas account for a considerable part. This design is based on the research of mine gas detection system based on AT89C51 single chip microcomputer.

This design is based on AT89C51 single-chip microcomputer, and combined with peripheral components such as methane sensor, A/D conversion chip, LCD liquid crystal display. A mine gas detection system for methane identification, threshold alarm and concentration display was designed. It converts information about the composition and concentration of the gas into electrical signals, which enables detection, monitoring, and alarming.

\section{Main problems in mine gas system}

The main component of the gas in the mine is methane, but it is also mixed with several other flammable and explosive gases. For methane, the limit of explosion is $3.5 \%$ to $16 \%$, that is, when the ratio of air to it is between $3.5 \%$ and $16 \%$, it can explode if exposed to an open flame. Therefore, in order to avoid this situation, it is necessary to monitor and limit the concentration of gas, and it is very important to control it in a timely manner [1]. In the current gas detection process, there are many remaining problems, for example, the life of the component is relatively short, and if the failure occurs, the detection result will be invalid, and it is easy to cause an accident; and the sensor will be affected by the mine environment, and the zero drift will occur. The phenomenon makes the sensor unable to work stably. Therefore, under the system of this kind of single chip, these problems are mainly solved. In this system, the accuracy and stability of the sensor have been greatly improved due to the effective use of modern MCU related processing technology [1]. In addition, due to the combination of PIC microcontroller hardware and software, not only the monitoring performance of the system is improved, but also the production cost of the system is reduced. 


\section{The mine gas monitoring system based on single chip}

\subsection{The working principle of single crystal mine gas monitoring system.}

The whole design system can be divided into six parts: reset circuit, clock circuit, AD conversion circuit, display circuit, alarm circuit and single chip microcomputer. In order to better illustrate the architecture of the design, it is specifically illustrated by a component diagram [2]. As shown in Figure 1 below.

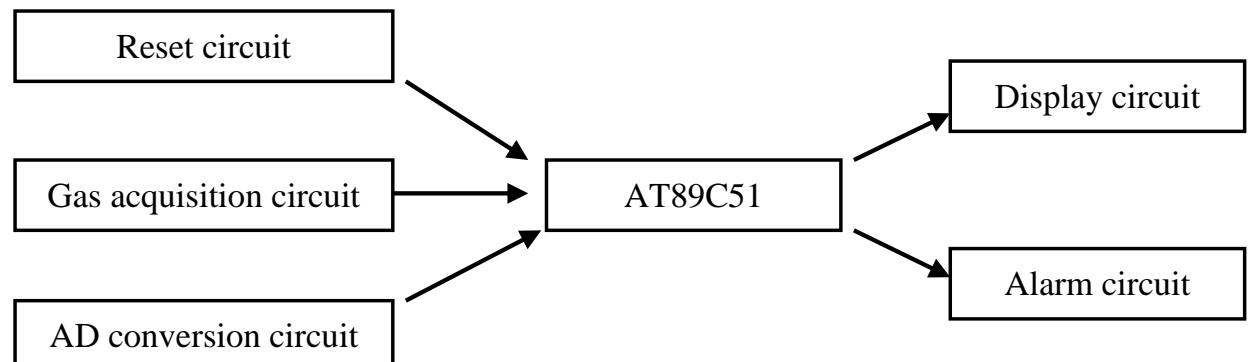

Fig.1 System structure diagram

Performance indicators of this design,

Display function: The concentration is displayed when gas is detected.

Gas concentration monitoring function: Using the analog system, the threshold is set to a certain value. When the gas concentration reaches this value, the buzzer sounds at the same time to light the staff signal.

Reset function: When the device fails or needs to be adjusted, press the reset button to initialize[3].

\subsection{Conversion chip selection.}

The A/D converter is a very important part of the monitoring system. It has many types, different performances and different pin functions. This design uses ADC0809, its pin pin diagram is shown in Figure 2-1, its resolution is eight bits. The ADC0809 has 8 channels of analog input lines (IN0 to IN7), which can perform A/D conversion on any channel under program control to obtain 8-bit binary digital quantities (D7 D0). The analog input section has 8 multiplexers, which can be selected by different combinations of 3-bit address inputs ADDA, ADDB, and ADDC. ALE is the address latch signal, which is active high, and latches the three address input signals. The main part is a successive approximation A/D conversion circuit, the internal circuit controlled by CLK works, START is the start command, the high level is valid, and the internal A/D conversion of ADC0809 is started [2]. When the conversion is completed, the output signal EOC is valid. OE is the output enable signal end, the active level is valid, the output tristate buffer is turned on, and the converted result is sent to the DB.

The CMOS monolithic successive approximation A/D converter used in this design is ADC0809, which consists of the following circuits, namely 8 analog switches, comparators, address latches and decoders, and 8-bit switches [2]. Tree D/A converter, 3-state output latch, successive approximation register, etc. Therefore, the A/D converter can handle eight analog inputs, and also has three-state output capability, which can be connected not only to various microprocessors, but also to work alone. Its input and output are compatible with TTL.

\subsection{Reset circuit.}

The reset circuit is one of the indispensable components in the operation of the microcontroller, and the microcontroller must be in a certain state when it is working. Uncertainty in port line level and input/output status may cause peripheral devices to malfunction, resulting in serious accidents; the contents of some internal control registers (special registers) may also cause timer overflow and the program has not started yet [3]. To interrupt and serial port transmission to send data to the peripheral.

The reset circuit designed by this design is shown in Figure 2. When the switch is not pressed, at the moment of power-on, since the voltage on the capacitor cannot be abrupt, the capacitor is in the charging (on) state, so the voltage on the RST pin and VCC the same. As the capacitor is charged, the 
voltage on the RST pin slowly drops [3]. By selecting a reasonable charging constant, it is guaranteed that the RST terminal has a high level of two machine cycles or more when the switch is pressed, thereby internally resetting the AT89C51.

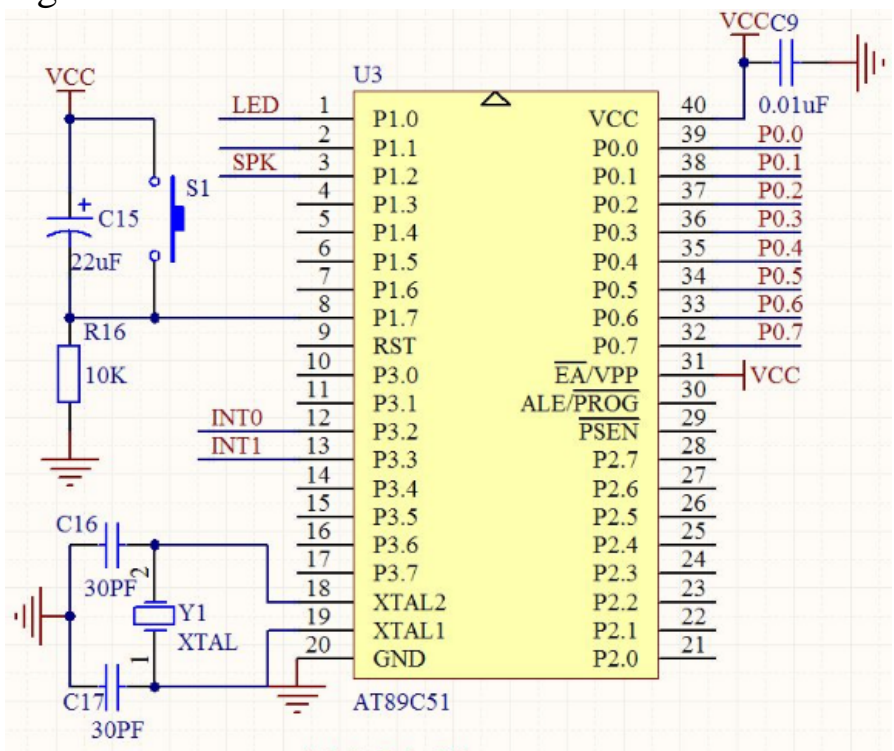

Fig 2. Reset circuit schematic

\subsection{Power circuit.}

Any circuit can not be separated from the power supply, the microcontroller is no exception. In this design, the AT89C51 single-chip microcomputer is designed as the core. It needs to be driven by the power supply circuit [4]. The power supply circuit is shown in Figure 3. J1 is the power supply socket, pin 1 is connected to $+5 \mathrm{~V}$, and pin 2 is grounded. D1 is the power indicator, R6 is the current limiting resistor, and $\mathrm{C} 2$ is the filter capacitor.

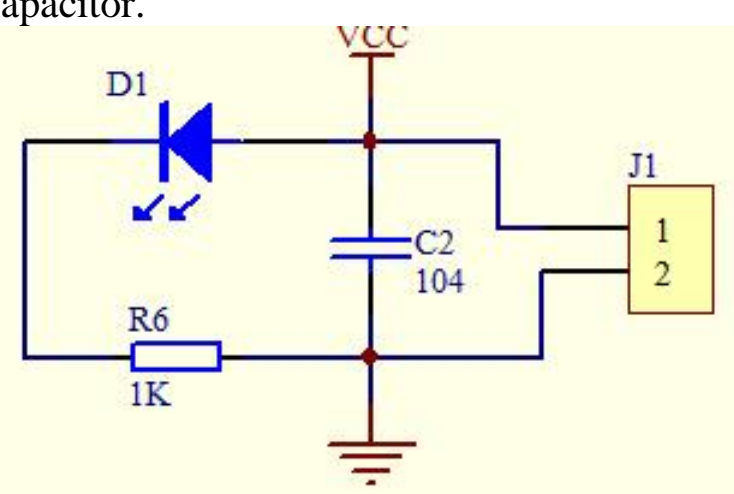

Fig.3 Power supply circuit schematic

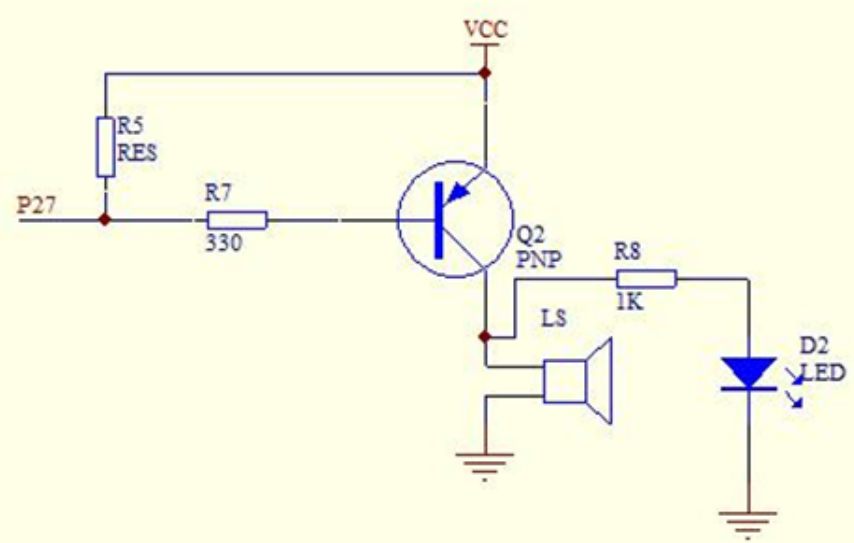

Fig.4 Alarm circuit schematic 


\subsection{Alarm circuit.}

The driving ability of the I/O of the MCU itself is not high, so the driver of the buzzer needs to add a PNP transistor, which can make the sound of the buzzer louder and play a better alarm function [4]. The alarm circuit is shown in Figure 4. When the gas concentration reaches the set value, the buzzer will alarm and the indicator light will illuminate.

\subsection{Sensor selection.}

Gas concentration detectors are classified into infrared type, optical interference type, sound speed difference type, gas sensitive semiconductor type, thermal conductivity type, and thermal catalytic type. Therefore, when carrying out a relatively specific measurement work, it is necessary to consider which kind of sensor is used, and it is necessary to take into consideration various factors before it can be determined. Since the same physical quantity is measured, sensors of various principles can also be selected [5]. When selecting a suitable sensor, consider the characteristics to be measured and the conditions of use of the selected sensor, such as the size of the sensor range; the volume of the sensor should be applied to the measured position; whether it is contact or non-contact measurement. After the analysis of the above problems, the design decided to use the MQ-4 sensor.

\subsection{Methane gas acquisition circuit.}

The gas concentration test part circuit is composed of a gas sensor MQ-4, and its function is to convert the volume fraction of gas gas into a corresponding analog voltage signal and output it [5].

The structure of the MQ-4 gas sensor consists of a miniature AL2O3 ceramic tube, a SnO2 sensitive layer, a measuring electrode and a heater, which are then fixed in a cavity made of plastic or stainless steel. The fabricated sensing element has 6 pins. Four of them are used for signal extraction and the remaining two are used to provide heating current.

\subsection{Display module selection.}

This design should choose the familiar LCD1062. Liquid crystal display (LCD), which can display text or display data using LCD1602. It is often used in course design, so everyone is no stranger to LCD monitors. The use of liquid crystal display as a display device in a single-chip mine gas detection system has the advantages of high quality, light weight, digital interface, small size, low power consumption, etc [6]. Therefore, LCD has gradually become an ideal display device for various portable electronic products. As seen in calculators, multimeters, electronic watches and many home electronics products, it is not only cheap, but also displays rich and beautiful content, which is a good replacement for LED digital tubes. The outline of the 1602 LCD is shown in Figure 5 below:

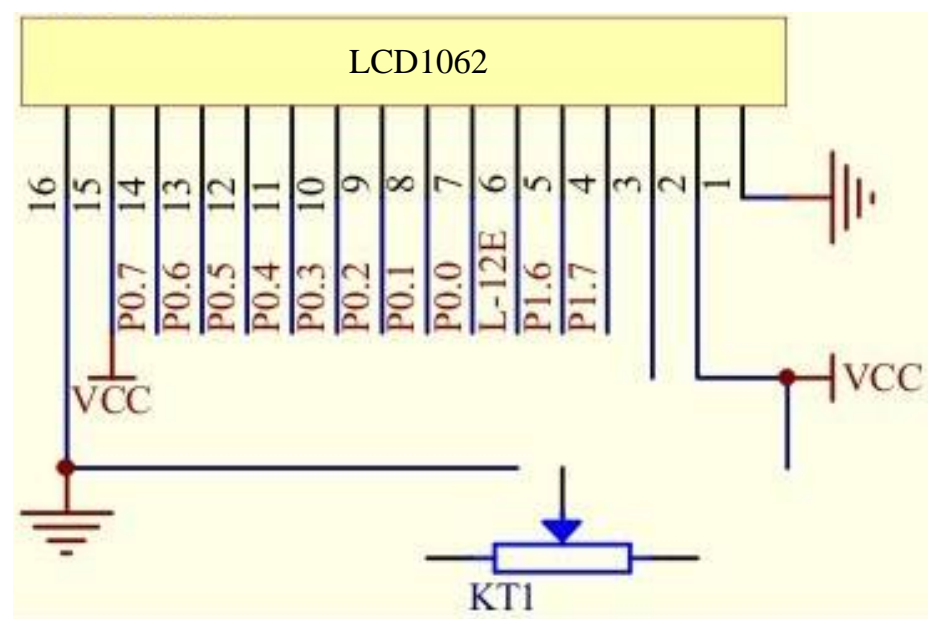

Fig.5 1602LCD

The principle of liquid crystal display is to use the physical characteristics of the liquid crystal to control the display area by voltage. As long as the required control voltage is input, the character can be displayed. 
The LCD is a slow display device, so be sure to read the LCD's busy state before writing each instruction. If the LCD is busy processing other instructions, wait; if not busy, execute the write command. To this end, the 1602 LCD has a busy flag bit BF, which is connected to D7 of 8-bit bidirectional data. If BF is low level " 0 ”, it means LCD is not busy; if BF is high level " 1 ”, it means LCD is busy and needs to wait. Assume that the 8-bit dual data line (D0 D7) of the 1602 LCD is transmitted through the P0 port of the MCU. As long as the P07 pin level of the P0 port is detected (D7 with P07), the busy flag bit BF can be known.

\section{Summary}

This MCU-based system allows the mine's gas to be monitored well, enabling early warning functions. The main feature of this system is that it is relatively simple in terms of hardware, and many hardware work is done under the operation of the software. Because the simple hardware can make the reliability and applicability of the system increase, the system is relatively comparative. stable. In addition, due to the combination of software and hardware, the development space of this system is still very large. In addition to the monitoring of gas, it can also develop the ventilation system and intelligent monitoring system of coal mines, which can be developed in the direction of multi-point jointing, so that the monitoring of gas Form a holistic, remote network control system with the control of the overall mine.

\section{Acknowledgement}

Foundation item: the topic is a funded project for the 2018 college-level key project of Jiangsu Vocational Institute of Commerce, Project name "Study on the Application of SCM Technology for Mine Gas Monitoring and Alarm system", Topic No.: JSJM011.

\section{References}

[1] H.X. Li, Application of Single Chip Microcomputer in Coal Mine Gas Monitoring and Early Warning, Beijing: Aerospace University Press, 2004, pp. 69-73.

[2] Y.Sh. Wu, Analysis of the application of information technology in coal mine gas monitoring and early warning, Electronic production, 2014, vol.3, pp.43-46.

[3] Q.H. Chen and Z.Y. Liu, Design of portable mine gas detection system, Shanxi Electronic Technology, 2011, vol.2, pp.21-24.

[4] Ch.M. Zhou, Analysis of the application of coal mine gas detection technology, Guangdong Science and Technology, 2012, vol.1, pp.16-18.

[5] D.H. Zhang, Discussion and Application of New Technology for Mine Gas Detection, Science and Technology Monograph, 2013, vol.10, pp. 352-352.

[6] Y.P. Bai, Research and analysis on new technology of mine gas monitoring, Science and Technology Guide, 2014, vol.21, pp.12-14. 